\title{
The Integration of Ink Art and Modern Advertising Design-- Analysis of the Effect of Ink Dots in Advertising Design in the Teaching of Art History
}

\author{
Jing Wang \\ (School of Literature, Xi'an University ,710065)
}

\begin{abstract}
Keywords: Ink dot; Art; Advertising design
\end{abstract}
\begin{abstract}
Ink dot, a form of ink art, is put to use in graphic design, bringing attractive design language expression which also full of personality characteristics. Analyzing ink technique in the teaching of art history and its performance and function in the modern advertising and explore the method of performance analysis of the ink point method, strengthened the students' ability of application, is an attempt at reform about course combined with the characteristics of the Advertising professional.

A brief history of art course for advertising majors is to provide certain artistic materials and references for advertising design. In the history of Oriental art, especially in the history of Chinese art, the representative ink art can bring people Oriental aesthetic interest. And when this kind of aesthetic feeling form is applied in modern design, it helps contemporary design build a kind of special Oriental charm. This paper takes the ink dot, a form of ink art that reflects the Oriental beauty, as the research object. Analyzed the ink dot and its application in modern design to help students build a bridge between the ink element in traditional art and modern advertising design, which is in order to be used in the future advertising professional design practice and innovate. Due to the limitation of space, the discussion scope of advertising design in this paper is graphic design.
\end{abstract}

\section{Analysis of Ink Spots in Eastern Ink Painting}

Ink art has many elements for reference and research, including ink color, line, artistic conception and so on. This paper mainly discusses the ink dot element and its value in advertising design. Because of its shape and the using characteristics of the Chinese brush, ink has a lot of change and appreciation, special effect and appreciate aesthetic feeling.

Pan Tianshou said, "the use of a Chinese brush for painting starts from one point. Though small in form, it is advisable to proceed with caution, and to write seriously, so that the picture may be neither more nor less. " It reveals the high level of Chinese painting's medium point modeling. In ancient Chinese painting theory, dots can be divided into 32 types, such as: “介” dot, “个” dot, chrysanthemum dot, pepper dot, etc. The dot can express the things in nature, such as: the dot in the tree for moss, the dot in the branches for flowers, etc. Dot can also be used to adjust thes black and white relationship of the picture, increase the warm atmosphere of the picture, help to form the rich spatial level of the picture, and adjust the beat rhythm of the line. In a word, the dot is useful. These "dots" are vertical and horizontal, long and short, large and small, round and flat, and upturned and hung. It is the flexible application of these different dots: the expansion of dots, the arrangement of dots, the aggregation and dispersion of dots, the combination of dots, etc. It shows the texture of the gods and rocks of natural objects appropriately, and increases the expression effect and beauty of the picture. Also, people like to talk about it in art history.

For example, the landscape painting from famous painter and calligrapher of the song dynasty $\mathrm{Mi} \mathrm{Fu}$ often use the boneless dot of the Chinese brush to show a soft moisture effect and reflects the dense scenery of jiangnan. The size, direction, arrangement and variation of dots have rich expressions. In his landscape paintings, the neat horizontal points on the mountain show the characteristics of the mountain and the sense of vegetation, while the dots on the tree with different sizes show the swaying appearance of the nearby leaves. As Huang Binhong said, "the dots can 
change in a thousand different ways, such as sowing seeds, dropping seeds into the ground, and growing fruits ....."

Of course, the dot in ink painting not only refers to the real point depicted by the Chinese brush, but also includes the virtual point formed by the blank, that is, the dot formed by the white space. Such virtual dots, although small in area, have an important role. In the air tight picture layout, it can adjust the visual feeling and the picture breath, giving the vision and psychology a breathing space. Meanwhile, it enriches the level of the picture and strengthens the sense of space, and also plays the role of echoing and balancing the picture.

\section{Analysis of Ink Dot in Advertising Design}

The dot in ink art, as well as the reflection, expansion and innovation of ink painting art on point, is applied in advertising design to bring Oriental expression and artistic conception to advertising design。

For example, Mr. Ikko tanaka designed a poster for Morisawa Font in 1996. In which there are eight different types of ink dots, which are free and explosive, like flowers blooming, like firecracker blasting, and like fire jumping. Such dots are passionate and represent thousands of different forms of font on paper. These ink dots fully show their own form and inner strength, both figurative and abstract. The ink dots from traditional paintings fully reflect their individuality, free and active, and full of emotions. With such a distinctive character of the ink dot to express the design creativity of the company, its strong break also reflects the designer's ingenuity.

Picture 1 is An Shangxiu's work whose shape is made of geometric squares with black appearance rules, but it has a strong flavor of ink. Through the arrangement of dots, a scene like snow and bamboo is formed, and the layers of black、 white and gray in the picture are also formed. The dots have the same shape and size seems to have formed a wonderful ink painting work through the ingenious combination and application of the designer. Because of different patterns and combinations of dots, the picture has formed a jumping sense which is new to people. The abstract application of the dots, out of the traditional brushwork, continuation of the look and feel of the ink dot, designers told the different understanding of ink painting techniques ussed in the design on another level.

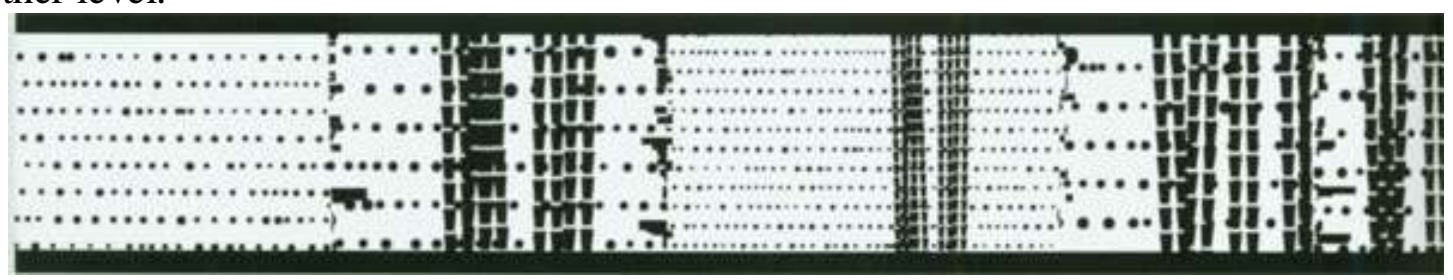

Figure 1 catalogue design (anshanxiu design)

Figure 2 is painted by Japanese designer Hiroyuki Ueno. The main element is still ink dots, which are slow and fluid in expression. The ink dot is like a hill, like a forest, like a speck offur. Ink dot has the grid constraint, which makes the ink point show a kind of rhythmic beauty in the flow, but it has the change of size and shape, and also has the active power. It feels like a flowing dune or a lush forest. Thick ink color and dense arrangement make people feel a strong visual oppression force, attract people for a long time.

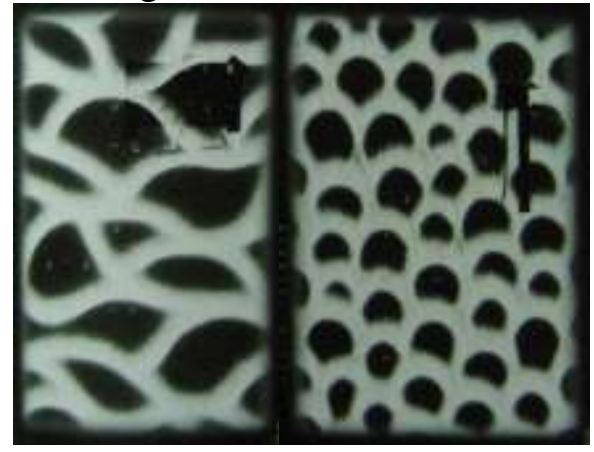


Figure 2. poster (designed by Hiroyuki Ueno)

For another example, Japanese designer Takaaki fujimoto's book design works, the cover design of the ink dot is very expressive. Most of the cover is in white background color, and is evenly distributed with the embossed title "AWAJI KAMA BOKO AKIYAMA". A small red brush stroke in the middle of the cover sets off the white author's name. But the most emotional part is not the raised text content, but on the title of the large and small ink dots. Falling from the top to the bottom, like bubbles, dewdrops, or like snow, became the most important part of the cover. These ink dots are the middle layer of the entire cover, adjusting the distance between the author's name and the title of the book, and it also increasing the three-dimensional space of the picture. It reveals the rich content of the book with kid gloves, which implies that the style of the book is warm and soft.

Also, Kan Tai-keung designed the posters concluding life, water, fire and wind. Most of the poster area are superimposed by the computer effects of ink dots and natural things. The pictures all with ink as the bottom rich in layers, and gradually to the upper layer of more specific leaves, rocks, water and gas. Ink and leaves, fire, wind and water that represent life combined naturally. Ink dots look like water, fog, an ancient mirror or an island. The life, water, fire and wind all from the ink points, and are linked together with ink, and may have a reciprocal transformation. The deep and light change and diffusion of the ink make people more associate, and it shows the author's Oriental philosophy well.

Kan Tai-keung is a designer with the characteristics of the eastern. His work has a lot of ink elements, and is very Oriental. His exhibition, "the years of ink," also used ink language. Below the white bottom is a black circular ink-stone, which is painted with a red brush shape dot. The design of this poster has its own new ideas, and used the comparison and substitution of the dots wonderfully. The dot and the plane are relative, the ink stone is the plane to the red figure, but it is a dot compare to the white paper. And then analyzes the inkstone as a dot from this angle. The ink of Kan Tai-keung is often changed into the ink stone, and there is ink in the inkstone, then the real ink shows a deep and stable effect, just as Mr Jin has been wearing a sword for decades. This dot is not ink, but it is better than ink, and there is a combination of people's thought. The inkstone is more like a thick ink lotus leaf, and the red dots above look like the flowers in the paintings. This flower makes it a lotus flower, which makes the ink stone has the weight of the balance. The ink is also live and the picture become vivid. The author uses the ink dot element lively, and the mean of the painting is integrated into different perspectives, so that people can get traditional feelings from new visual elements.

In addition, Kan Tai-keung's poster for a festival event in a Japanese town also uses the ink dot element well. The whole picture is full of a child holding up his hands by using paper-cutting techniques, which has the white child on red background. The most charming part is the child's face and mouth, using ink to draw a smiling mouth. Using the happy child to show the atmosphere of the festival, the theme of paper-cutting is the festival, and the smile of the paper cutting is replaced by the brushed one. And the point of laughter is the smile painted with the ink.

The part of the brush forms a focus, that is, the visual center of the picture, and shows the emotion of the picture. "The point is the center of force. When there is only one dot in the picture, people's sights are all at this dot. It is tense. And it has the effect of tension in the space. "Because of the unique feeling of the ink, the mouth was a pretty dot differs from other figures in the poster. The changable feeling characteristic of the ink brings sights easily in the poster. Ink has the characteristic of change rich, active and vivid, it can come out naturally from the regular paper-cut. And make people pay attention to this vivid and lively dot unconsciously. Meanwhile, this point is light, warm and relaxed, full of the implicit joy of the eastern. That makes it become the window for the characters to express their feelings in the picture. The emotion conveyed by this dot is longed to the whole body of child through the laughter, and also to the eyes and hearts of the viewer.

Ink dot element is a good way for designers to show images and emotions. The layers, the rich forms and the psychological level of the feelings of the ink dot all bring people different appreciation of beauty. Using such points in advertising design can make the work more charming. 


\section{Analysis of Ink Dot Application in Advertisement}

The ink dot in advertisement design has rich expression because of its changeable form and rich color level. At the same time, the recognition of the ink dot makes it naturally have the Oriental aesthetic taste and form a picture with the Oriental aesthetic feeling, that is the reason why it is popular. The effect of advertising design using ink dot elements may not satisfactory. So it is necessary to analyze and summarize the use of ink dot elements so as to get better effects.

First of all, the use of ink dot should have relation with the theme, that is, the expression content and traditional ink. Such as emphasis on culture and traditional ones is better to use ink point element to express: the traditional festivals, local tourism advertising or traditional goods such as souvenir and food. It is proper to use ink elements.

Next, ink dot elements are benefit to express different artistic conception. The combination of the ink color and the brush makes the ink point change a thousand times and shows a great expression space, which makes the picture show different artistic conception. For example, the warm mood, the hard and cold mood, with a sense of speed of storm and rain, and so on. Different ways of using the brush and more or less water in the brush can show different ink patterns, create different artistic conception.

Finally, the creative use of the ink dot element can show unexpected effects. Ink dot elements are generally connected with traditional and Chinese art, but they still have their modern sense and more expressive methods. Firstly, the traditional ink dot can use modern color language, or combine with modern graphics to form a contrast and complementary effect. Secondly, modern elements can be expressed in the language of design to show the charm of traditional ink dots, such as the design of an shangxiu mentioned in the article. The creative use of ink dot elements leaves people a deep impression.

Study the application of ink in advertising design further and analyse the case,put forward the use of ink element method from the art history teaching has positive influence to the art of teaching the brief history of art in advertising. A better understanding of ink art and its application in the modern advertising design can help advertising professional students use it in practice better. It is a try for course innovation according to the actual needs of professional. This paper is one of the achievements of the 2018 key course project "brief history of art" (project number KGB201808) of xi 'an university.

\section{References}

[1] He Jie, kan tai-teung, Hang Jian. Between the Chinese traditional graphic and modern visual design portfolio - poetic age - the design of the poetic (Chinese traditional graphic and modern visual design portfolio) [M]. Liaoning, liaoning art publishing house, 2007.

[2] Li Jiansheng. Artistic and cultural interpretation of contemporary design [M]. Henan: henan fine arts press, 2002.

[3] Wang Xu. Design process of anshangxiu graphic designer [M]. Beijing: China youth publishing house, 1997.

[4] Yang Renkai. Chinese calligraphy and painting [M]. Shanghai: Shanghai ancient books press, 1990.

[5] Zhao Dianze. Composition art [M]. Liaoning: liaoning fine arts press, 1987.

[6]Julius Wiedemann. Contemporary Japanese graphic design [M]. Japan: Ute Wachendort, 2004. 